\title{
Base-modified thymidine and thymine analogs with low cytotoxicity effectively obstruct DNA replication in papovaviridae
}

Kayla M. Borland, Patrick R. Wolfkiel, Matthew P. Burke, Sean M. Lawson, Courtney A. Stockman, Aron P. Bercz, Julia N. Tolstolutskaya and Vladislav A. Litosh*

*Correspondence: Vladislav.Litosh@uc.edu

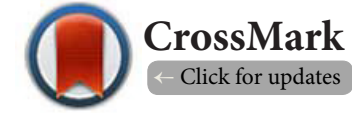

Department of Chemistry, University of Cincinnati, 301 W. Clifton Ct. Cincinnati, OH 45221, USA.

\begin{abstract}
Background: Current chemotherapeutic antimetabolites often exhibit severe side effects that limit their use as drugs; therefore, we designed nucleoside compounds with mechanisms of action focusing on inhibiting DNA replication rather than targeting multiple pathways. We previously discovered cytotoxic basemodified thymidine and thymine analogs that show higher selectivity against cancerous versus normal cells compared to the current antimetabolites used in cancer chemotherapy. We anticipated these antimetabolites have the potential to effectively inhibit viral DNA replication while showing low cytotoxicity.

Methods: Base-modified thymidine and thymine analogs were synthesized and their anti-viral activity was evaluated in human cells infected with human pappiloma, John Cunningham, and BK viruses using quantitative DNA polymerase chain reaction assay. In addition, their toxicity toward host cells was determined using CellTiter-Glo assay, and compared to cytotoxicity toward human breast cancer cells.

Results: Novel lead compounds with high activity against human papilloma (HPV) and John Cunningham $(\mathrm{JCV})$ viruses have been identified. Their EC50 values lie in low micromolar range (1-2 $\mu \mathrm{M})$, which is significantly less than that of cidofovir $(9-10 \mu \mathrm{M})$, a current drug used against DNA viruses. Cytotoxicity of the leads toward the host cells was found to be in 200-300 $\mu \mathrm{M}$ range, which is generally higher than that observed toward MCF-7 human breast cancer cells. None of the tested compounds significantly inhibited BK viral DNA replication.

Conclusion: The lead compounds affect the viruses substantially more selectively than the host cells, which makes them a novel class of bioactive compounds with the potential to become effective anti-viral drugs.
\end{abstract}

Keywords: Nucleosides, antimetabolites, DNA replication, anti-viral agents, anti-cancer agents, human papillomavirus, John Cunningham virus, breast cancer, chemotherapeutics

\section{Introduction}

Papovaviridae is a family of DNA viruses that are associated with serious diseases in patients with compromised immune system. Of those, human papillomavirus (HPV) is the most common sexually transmitted infection worldwide [1]. The high risk types HPV (e.g., 16,18) cause cervical cancer [2-4], while the low risk types HPV (e.g., 6,11) incur respiratory diseases, sometimes fatal, in spite of the current treatment [5]. John Cunningham virus (JCV) is known to cause progressive multifocal leukoencephalopathy [6], which is usually deadly. BK virus, a close analog of JCV, is implicated in nephropathy [7] in renal transplant patients. Currently, there are no effective drugs that inhibit or cure these viral infections without off-target toxicity.
Therefore, developing novel therapeutic agents against these viruses with low cytotoxicity can be lifesaving.

In the past half century, modified nucleobase and nucleoside analogs [8], otherwise termed antimetabolites, have substantially impacted treatment of cancer [9] and infectious diseases $[10,11]$. Physiologically, they readily undergo cellular uptake by nucleoside transporters [12], followed by metabolism into nucleotides [11], the active species capable of affecting a number of intracellular targets, such as enzymes producing nucleic acids [13-15] and single nucleotides [16,17], and targeting mitochondria leading to apoptosis [18]. Incorporation of antimetabolites into DNA results in damage signaling [19], obstruction of DNA synthesis [20] and repair [21]. Although inhibition of viral DNA 
replication without affecting the host DNA is more likely [22] than selective targeting of neoplastic DNA, occasionally, antimetabolite anti-viral agents do incur severe side effects, such as hepatotoxicity [23], hematopoietic toxicity [24], myelosuppression [25], hyperlactatemia, and lactic acidosis [26].

Recently, we have discovered base-modified thymidine analogs that show higher cytotoxicity toward cancerous cells compared to benign [27]. Although the modifying moiety attached to the nucleobase obstructs further DNA synthesis upon incorporation of the 5 '-triphosphate of such nucleoside into a partial double helix DNA primer [28], the preliminary insight into the mechanism of action strongly suggested inhibition of a DNA polymerase instead [27]. However, it was still evident that termination of DNA synthesis occurs only in the presence of a certain bulky group attached at the 5-methyl group of the thymine nucleobase. Considering the high selectivity of the novel species toward cancer cells versus normal cells, we have decided to investigate the possibility of selective targeting of viral DNA replication versus killing the host cell by the newly developed nucleoside analogs. In this paper, we report the evaluation of 16 thymidine and 15 thymine analogs (Figure 1) with modifications at the 5-methyl group in their ability to obstruct DNA replication of HPV and JC viruses, and compare the antiviral activity to the toxicity toward the host cells. The lead compounds identified from these structure-activity relationship studies for both viruses exhibit diminutive cytotoxicity, which opens the new passage to further drug development. Our contribution to the field of anti-viral drug discovery is significant as it gives the promise to access new agents for these viral diseases that currently have no non-toxic drugs available for treatment.

$\mathrm{R}=\mathrm{Br}(\mathbf{1})$ or $\mathrm{Cl}(\mathbf{2}) ; \mathrm{R}_{1}, \mathrm{R}_{2}=\mathrm{Alk}$ or $\mathrm{Ar} ; \mathrm{R}_{3}=\mathrm{H}(\mathbf{2})$ or
$\mathrm{R}_{4}=\mathrm{H}(\mathbf{2})$ or $\mathrm{Boc}(\mathbf{1}) ; \mathrm{R}_{5}=\mathrm{H}$ (3a-18a) or $\mathrm{TBS}$ (1)
Figure 1. Synthesis of 5-modified thymines and thymidines:
(i) appropriate alcohol, neat, 110-140 $0^{\circ} \mathrm{C}, 0.5-3 \mathrm{~h}$; (ii) (for a)

\section{Materials and methods Synthesis}

All chemicals, reagents, and solvents were purchased from Sigma-Aldrich Inc., $\mathrm{TCl}$, and Fisher Scientific, Inc., and used as received unless stated otherwise. All reactions were carried out under an atmosphere of dry argon in oven-dried glassware. Indicated reaction temperatures refer to those of the reaction bath, while room temperature $(r t)$ is noted as $25^{\circ} \mathrm{C}$. Pure reaction products were typically dried under high vacuum in the presence of phosphorus $(\mathrm{V})$ oxide. Analytical thin layer chromatography (TLC) was performed using glass backed silica plates $(5 \times 20 \mathrm{~cm}, 60 \AA, 250 \mu \mathrm{m})$. Visualization was accomplished using a $254 \mathrm{~nm}$ UV lamp. ${ }^{1} \mathrm{H}$ and ${ }^{13} \mathrm{C}$ NMR spectra were recorded on either a Bruker Avance $400 \mathrm{MHz}$ or Bruker DPX $500 \mathrm{MHz}$ spectrometer using solutions of samples in either of the deuterated solvents: DMSO, methanol, acetonitrile. Chemical shifts are reported in ppm with tetramethylsilane as standard. Data are reported as follows: chemical shift, number of protons, multiplicity ( $s=$ singlet, $d=$ doublet, $d d=$ doublet of doublet, $\mathrm{t}=$ triplet, $\mathrm{q}=$ quartet, $\mathrm{b}=\mathrm{broad}, \mathrm{m}=$ multiplet, $\mathrm{abq}=\mathrm{ab}$ quartet), and coupling constants. High resolution mass spectral data were recorded on a Shimadzu Q-TOF 6500 instrument. All novel compounds were characterized by ${ }^{1} \mathrm{H},{ }^{13} \mathrm{C}, \mathrm{DEPT}{ }^{13} \mathrm{C}$ NMR spectroscopy and high resolution mass spectrometry. The identity of previously made nucleoside derivatives was confirmed by comparison of their ${ }^{1} \mathrm{H}$ NMR to the published data (reference provided). HPLC analysis of final products was performed on an Agilent 1200 HPLC with UV detection. Compounds biologically tested were at least $95 \%$ pure as judged by ${ }^{1} \mathrm{H}$ NMR and HPLC.

\section{General procedure for preparation of base-modified thymidines}

5-Bromomethyl-3-N-(tert-butyloxy)carbonyl-3', 5'-bis-0(tert-butyl) dimethylsilyl-2'-deoxyuridine (1) [28] and appropriate alcohol (4-20 eq.) were heated neat at $110-120^{\circ} \mathrm{C}$ for the period between 15 minutes to 2 hours under argon atmosphere. The mixture was cooled down to room temperature, dissolved in tetrahydrofuran $(c a 5 \mathrm{ml})$, and to this solution chilled at $0^{\circ} \mathrm{C}$ tetra-n-butylammonium fluoride trihydrate (TBAF) was added (ca 2.5 eq.). The reaction mixture was stirred for 2 hours while gradually warming up to room temperature. The solvent was removed under reduced pressure and the residue was purified by silica gel (chloroform/methanol=1:0 to 10:1) and then by $\mathrm{C} 8$ reverse-phase column chromatography (water/methanol=19:1 to 1:4) to yield the product as a waxy solid. Compounds 3a11a and 14a-18a were obtained as reported previously [27].

5-[1-(phenyl)-1-hexyloxymethyl]-2'-deoxyuridine (12a) Heating 1 (103mg, $0.158 \mathrm{mmol}$ ) with a-n-hexylbenzyl alcohol (1-phenyl-1-hexanol, $376 \mathrm{mg}, 1.580 \mathrm{mmol}$ ) for 2 hours at $116^{\circ} \mathrm{C}$ followed by purification of bis- and mono-TBS products with subsequent treatment with TBAF $(161 \mathrm{mg}, 0.457 \mathrm{mmol})$ afforded after purification $18 \mathrm{mg}(27 \%)$ of product as 1:1 mixture of diastereomers. ${ }^{1} H N M R\left(400 \mathrm{MHz}, \mathrm{CD}_{3} \mathrm{OD}\right)$ for diastereomers: $\delta$ $7.96(\mathrm{~s}, 1 \mathrm{H}), 7.31(\mathrm{~m}, 5 \mathrm{H}), 6.28(\mathrm{~m}, 1 \mathrm{H}), 4.42(\mathrm{~m}, 1 \mathrm{H}), 4.36(\mathrm{~m}$, $1 \mathrm{H}), 4.11(\mathrm{~m}, 2 \mathrm{H}), 3.95(\mathrm{~m}, 1 \mathrm{H}), 3.80(\mathrm{AB} \mathrm{d}, 1 \mathrm{H}, J=12.0 \mathrm{~Hz})$, $3.75(\mathrm{AB} \mathrm{d}, 1 \mathrm{H}, \mathrm{J}=12.0 \mathrm{~Hz}), 2.28(\mathrm{~m}, 1 \mathrm{H}), 2.21(\mathrm{~m}, 1 \mathrm{H}), 1.82(\mathrm{~m}$, $1 \mathrm{H}), 1.62(\mathrm{~m}, 1 \mathrm{H}), 1.28(\mathrm{~m}, 8 \mathrm{H}), 0.89(\mathrm{~m}, 3 \mathrm{H}) .{ }^{13} \mathrm{C} \mathrm{NMR}(100$ $\mathrm{MHz}, \mathrm{CD} \mathrm{OD}_{3}$ ) for diastereomers $\delta 163.65$ (C), 150.69 (C), 142.52 (C), $139.21(\mathrm{CH}), 120.05(\mathrm{CH}), 127.17(\mathrm{CH}), 126.49$ and 126.43 
$(\mathrm{CH}), 111.38(\mathrm{C}), 87.56(\mathrm{CH}), 85.16$ and $85.09(\mathrm{CH}), 82.11$ and $82.00(\mathrm{CH}), 70.91$ and $70.85(\mathrm{CH}), 63.11$ and $62.91\left(\mathrm{CH}_{2}\right), 61.50$ $\left(\mathrm{CH}_{2}\right), 39.99\left(\mathrm{CH}_{2}\right), 37.89$ and $37.86\left(\mathrm{CH}_{2}\right), 31.58\left(\mathrm{CH}_{2}\right), 28.89$ $\left(\mathrm{CH}_{2}\right), 25.44$ and $25.39\left(\mathrm{CH}_{2}\right), 22.27\left(\mathrm{CH}_{2}\right), 13.04\left(\mathrm{CH}_{3}\right)$. HRMS (ESI) for [MNa] ${ }^{+}$calculated: 455.21581 , observed: 455.21523 .

\section{5-[1-(2-nitro)phenyl-1-hepthoxymethyl]-2'-deoxyuridine (13a)}

Heating 1 (97mg, 0.149mmol) with a-hexyl-2-nitrobenzyl alcohol (1-(2-nitro)phenyl-1-heptanol) (142mg, $0.597 \mathrm{mmol}$ ) for 15 minutes at $112-114^{\circ} \mathrm{C}$ followed by purification of bis- and mono-TBS products with subsequent treatment with TBAF $(48 \mathrm{mg}, 0.150 \mathrm{mmol})$ afforded after purification $18 \mathrm{mg}(25 \%)$ of product as 1:1 mixture of diastereomers. ${ }^{1} \mathrm{H} N \mathrm{NMR}(400 \mathrm{MHz}$, $\left.C D_{3} \mathrm{OD}\right)$ for diastereomers: $\delta 8.02$ and $7.98(2 \mathrm{~s}, 1 \mathrm{H}), 7.92(\mathrm{~d}$, $J=8.4 \mathrm{~Hz}, 1 \mathrm{H}), 7.80(\mathrm{~d}, J=8.0 \mathrm{~Hz}, 1 \mathrm{H}), 7.69(\mathrm{~m}, 1 \mathrm{H}), 7.48(\mathrm{~m}, 1 \mathrm{H})$, $6.25(\mathrm{~m}, 1 \mathrm{H}), 4.40(\mathrm{~m}, 1 \mathrm{H}), 4.09(\mathrm{~m}, 3 \mathrm{H}), 3.92(\mathrm{~m}, 1 \mathrm{H}), 3.74(\mathrm{~m}$ $2 \mathrm{H}), 2.24(\mathrm{~m}, 2 \mathrm{H}), 1.70(\mathrm{~m}, 2 \mathrm{H}), 1.47(\mathrm{~m}, 4 \mathrm{H}), 0.92(\mathrm{~m}, 7 \mathrm{H}) .{ }^{13} \mathrm{C}$ NMR $\left(100 \mathrm{MHz}, \mathrm{CD}_{3} \mathrm{OD}\right)$ for diastereomers $\delta 165.09$ (C), 150.00 and $149.98(\mathrm{C}), 141.34$ and $141.22(\mathrm{CH}), 139.82(\mathrm{C}), 134.50(\mathrm{CH})$, 129.60 and $129.56(\mathrm{CH}), 129.34(\mathrm{CH}), 125.23(\mathrm{C}), 125.17(\mathrm{CH})$, $112.38(\mathrm{C}), 89.03(\mathrm{CH}), 86.50(\mathrm{CH}), 78.19$ and $77.46(\mathrm{CH}), 72.32$ and $72.77(\mathrm{CH}), 65.29$ and $65.11\left(\mathrm{CH}_{2}\right), 62.86\left(\mathrm{CH}_{2}\right), 41.47$ and $41.40\left(\mathrm{CH}_{2}\right), 39.16\left(\mathrm{CH}_{2}\right), 33.10$ and $32.97\left(\mathrm{CH}_{2}\right), 30.81$ and 30.49 $\left(\mathrm{CH}_{2}\right), 27.08\left(\mathrm{CH}_{2}\right), 23.77$ and $23.72\left(\mathrm{CH}_{2}\right), 14.45\left(\mathrm{CH}_{3}\right)$. HRMS (ESI) for [MNa] ${ }^{+}$calculated: 500.20088, observed: 500.20032.

General procedure for the synthesis of modified thymines 5-Chloromethyluracil (2) and appropriate alcohol (4-11 eq.) were heated neat at $120^{\circ} \mathrm{C}$ for 0.5 to 3 hours under argon atmosphere. The mixture was cooled down to room temperature, dissolved in dichloromethane/methanol=20:1, and silica (ca $5 \mathrm{~g}$ ) was added. The solvent was removed under reduced pressure and the solid was applied onto a silica gel column. Chromatography $\left(\mathrm{SiO}_{2^{\prime}} \mathrm{CH}_{2} \mathrm{Cl}_{2} / \mathrm{MeOH}=20: 1\right)$ afforded the product as a powder.

\section{5-[1-phenyl-2,2-(dimethyl) propoxymethyl] uracil (3b) Treatment of $2(57 \mathrm{mg}, 0.355 \mathrm{mmol})$ with $660 \mathrm{mg}(4.018 \mathrm{mmol})$ of commercial racemic a-tert-butylbenzyl alcohol (2,2-dimethyl- 1-phenyl-1-propanol) for 1 hour afforded after purification 25 mg of product (24\%). ${ }^{1} H N M R(400 \mathrm{MHz}, \mathrm{DMSO}-\mathrm{d} 6) \delta 11.09$ (br. $\mathrm{s}, 1 \mathrm{H}, \mathrm{D}_{2} \mathrm{O}$ exchangeable) 10.80 (br. $\mathrm{s}, 1 \mathrm{H}, \mathrm{D}_{2} \mathrm{O}$ exchangeable), $7.31(\mathrm{~m}, 6 \mathrm{H}), 4.05(\mathrm{~s}, 1 \mathrm{H}), 3.97(\mathrm{AB} \mathrm{d}, 2 \mathrm{H}, J=12.1 \mathrm{~Hz}), 3.82(\mathrm{AB} \mathrm{d}$, $2 \mathrm{H}, \mathrm{J}=12.1 \mathrm{~Hz}), 0.82(\mathrm{~s}, 9 \mathrm{H}) .{ }^{13} \mathrm{CNMR}\left(100 \mathrm{MHz}, \mathrm{CD}_{3} \mathrm{OD}\right) \delta 164.05$ (C), $151.71(\mathrm{C}), 140.06(\mathrm{CH}), 139.73(\mathrm{C}), 128.58(\mathrm{CH}), 127.93$ $(\mathrm{CH}), 127.62(\mathrm{CH}), 109.90(\mathrm{C}), 88.61(\mathrm{CH}), 63.73\left(\mathrm{CH}_{2}\right), 35.60$ (C), $26.52\left(\mathrm{CH}_{3}\right)$. HRMS (ESI) for [MNa] ${ }^{+}$calculated: 311.13661, observed: 311.13661.}

\section{5-[1-(2-nitrophenyl)-2,2-(dimethyl)propoxymethyl] uracil (4b)}

Treatment of $2(50 \mathrm{mg}, 0.311 \mathrm{mmol})$ with $272 \mathrm{mg}(1.3 \mathrm{mmol})$ of racemic a-tert-butyl-2-nitrobenzyl alcohol (2,2-dimethyl1-(2-nitrophenyl)-1-propanol) for 2.5 hours afforded after purification $32 \mathrm{mg}$ of product (31\%). 'H NMR $(400 \mathrm{MHz}$, DMSOd6) $\delta 11.09$ (br. s, $1 \mathrm{H}, \mathrm{D}_{2} \mathrm{O}$ exchangeable) 11.09 (br. s, $1 \mathrm{H}, \mathrm{D}_{2} \mathrm{O}$ exchangeable), $7.88(\mathrm{~d}, 1 \mathrm{H}, J=7.9 \mathrm{~Hz}), 7.70(\mathrm{~m}, 2 \mathrm{H}), 7.56(\mathrm{t}, 1$ $\mathrm{H}, J=7.4 \mathrm{~Hz}), 7.41(\mathrm{~s}, 1 \mathrm{H}), 4.79(\mathrm{~s}, 1 \mathrm{H}), 4.08(\mathrm{AB} \mathrm{d}, 2 \mathrm{H}, J=11.6$ $\mathrm{Hz}), 3.94(\mathrm{AB} \mathrm{d}, 2 \mathrm{H}, J=11.6 \mathrm{~Hz}), 0.79(\mathrm{~s}, 9 \mathrm{H}) .{ }^{13} \mathrm{CNMR}(100 \mathrm{MHz}$, $\left.C D_{3} \mathrm{OD}\right) \delta 160.04(\mathrm{C}), 151.73(\mathrm{C}), 150.85(\mathrm{C}), 141.28(\mathrm{CH}), 133.65$ (C), $132.76(\mathrm{CH}), 130.08(\mathrm{CH}), 129.17(\mathrm{CH}), 124.22(\mathrm{CH}), 109.04$ (C), $80.96(\mathrm{CH}), 64.42\left(\mathrm{CH}_{2}\right), 36.53(\mathrm{C}), 25.97\left(\mathrm{CH}_{3}\right)$. HRMS (ESI) for $[\mathrm{MH}]^{+}$calculated: 334.13975 , observed: 334.13977 ; for

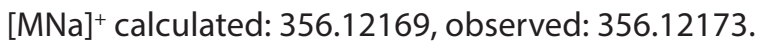

\section{5-[1-(2-methoxyphenyl)-2,2-(dimethyl)propoxymethyl] uracil (5b)}

Treatment of $2(50 \mathrm{mg}, 0.311 \mathrm{mmol})$ with $290 \mathrm{mg}(1.5 \mathrm{mmol})$ of racemic a-tert-butyl-2-methoxybenzyl alcohol (2,2-dimethyl1-(2-methoxyphenyl)-1-propanol) for 1 hour afforded after purification $20 \mathrm{mg}$ of product (20\%). ' $\mathrm{H} N \mathrm{NMR}(400 \mathrm{MHz}$, DMSOd6) $\delta 11.05$ (br. s, $1 \mathrm{H}, \mathrm{D}_{2} \mathrm{O}$ exchangeable) 10.78 (br. s, $1 \mathrm{H}, \mathrm{D}_{2} \mathrm{O}$ exchangeable), $7.25(\mathrm{~m}, 3 \mathrm{H}), 6.96(\mathrm{~m}, 2 \mathrm{H}), 4.52(\mathrm{~s}, 1 \mathrm{H}), 3.98$ (d, $1 \mathrm{H}, J=12.0 \mathrm{~Hz}), 3.77(\mathrm{~m}, 4 \mathrm{H}), 0.83(\mathrm{~s}, 9 \mathrm{H})$. HRMS (ESI) for [MNa] ${ }^{+}$calculated: 341.14718 , observed: 341.14723 ; for [M$\mathrm{H}^{-}$calculated: 317.15068 , observed: 317.15058 .

\section{5-[1-(3-methoxyphenyl)-2,2-(dimethyl)propoxymethyl] uracil (6b)}

Treatment of $2(50 \mathrm{mg}, 0.311 \mathrm{mmol})$ with $190 \mathrm{mg}(1.0 \mathrm{mmol})$ of racemic a-tert-butyl-3-methoxybenzyl alcohol (2,2-dimethyl1-(3-methoxyphenyl)-1-propanol) for 1 hour afforded after purification $33 \mathrm{mg}$ of product (33\%). ' $\mathrm{H} N \mathrm{NMR}(400 \mathrm{MHz}$, DMSOd6) $\delta 11.08$ (br. s, $1 \mathrm{H}, \mathrm{D}_{2} \mathrm{O}$ exchangeable) 10.64 (br. s, $1 \mathrm{H}, \mathrm{D}_{2} \mathrm{O}$ exchangeable), $6.77(\mathrm{~m}, 4 \mathrm{H}), 5.11(\mathrm{~m}, 1 \mathrm{H}), 4.44(\mathrm{~s}, 1 \mathrm{H}), 4.20$ $(\mathrm{m}, 1 \mathrm{H}), 3.73(\mathrm{~m}, 4 \mathrm{H}), 0.88(\mathrm{~s}, 9 \mathrm{H}) .{ }^{13} \mathrm{CNMR}\left(100 \mathrm{MHz}, \mathrm{CD}_{3} \mathrm{OD}\right)$ $\delta 164.29(\mathrm{C}), 157.02(\mathrm{C}), 151.20(\mathrm{C}), 143.15(\mathrm{CH}), 138.46(\mathrm{C})$, $130.25(\mathrm{CH}), 128.27(\mathrm{CH}), 113.84(\mathrm{CH}), 112.15(\mathrm{CH}), 112.05$ (C), $74.53(\mathrm{CH}), 65.94\left(\mathrm{CH}_{2}\right), 54.79\left(\mathrm{CH}_{3}\right), 36.87(\mathrm{C}), 26.16\left(\mathrm{CH}_{3}\right)$.

\section{5-[1-(4-methoxyphenyl)-2,2-(dimethyl)propoxymethyl]} uracil (7b)

Treatment of 2 (50mg, $0.311 \mathrm{mmol})$ with $252 \mathrm{mg}(1.3 \mathrm{mmol})$ of racemic a-tert-butyl-4-methoxybenzyl alcohol (2,2-dimethyl1-(4-methoxyphenyl)-1-propanol) for 2 hours afforded after purification $30 \mathrm{mg}$ of product (31\%). ${ }^{1} \mathrm{HNMR}\left(400 \mathrm{MHz}, \mathrm{CD}_{3} \mathrm{CN}\right)$ $\delta 8.86$ (br. s, $2 \mathrm{H}) 7.20(\mathrm{~m}, 2 \mathrm{H}), 6.89(\mathrm{~m}, 2 \mathrm{H}), 4.00(\mathrm{~m}, 1 \mathrm{H})$, $3.80(\mathrm{~m}, 4 \mathrm{H}), 0.84(\mathrm{~s}, 9 \mathrm{H}) .{ }^{13} \mathrm{CNMR}\left(100 \mathrm{MHz}, \mathrm{CD}_{3} \mathrm{CN}\right) \delta 167.11$ (C), $159.84(\mathrm{C}), 139.76(\mathrm{CH}), 130.48(\mathrm{CH}), 119.27(\mathrm{C}), 113.78$ $(\mathrm{CH}), 111.32(\mathrm{C}), 89.43(\mathrm{CH}), 64.08\left(\mathrm{CH}_{2}\right), 55.80\left(\mathrm{CH}_{3}\right), 36.21$ (C), $26.52\left(\mathrm{CH}_{3}\right)$. HRMS (ESI) for [MNa] $]^{+}$calculated: 341.14718, observed: 341.14724; for [M-H] calculated: 317.15068, observed: 317.15055 .

\section{5-[1-(2-bromophenyl)-2,2-(dimethyl)propoxymethyl] uracil (9b)}

Treatment of $2(50 \mathrm{mg}, 0.311 \mathrm{mmol})$ with $134 \mathrm{mg}(0.55 \mathrm{mmol})$ of racemic a-tert-butyl-2-bromobenzyl alcohol (2,2-dime- 
Borland et al. Biochemical Compounds 2016,

http://www.hoajonline.com/journals/pdf/2052-9341-4-3.pdf

doi: 10.7243/2052-9341-4-3

thyl-1-(2-bromophenyl)-1-propanol) for 2 hours afforded after purification $22 \mathrm{mg}$ of product (19\%). ${ }^{1} \mathrm{H} \mathrm{NMR}(400 \mathrm{MHz}$ DMSO-d6) $\delta 11.06$ (br. s, 1 H, $\mathrm{D}_{2} \mathrm{O}$ exchangeable) 10.82 (br. s, $1 \mathrm{H}, \mathrm{D}_{2} \mathrm{O}$ exchangeable), $7.59(\mathrm{~d}, 1 \mathrm{H}, J=8.0 \mathrm{~Hz}), 7.30(\mathrm{~m}, 4 \mathrm{H})$, $4.50(\mathrm{~s}, 1 \mathrm{H}), 3.92(\mathrm{~d}, 1 \mathrm{H}, J=12.0 \mathrm{~Hz}), 3.78(\mathrm{~d}, 1 \mathrm{H}, J=12.0 \mathrm{~Hz})$, $0.88(\mathrm{~s}, 9 \mathrm{H}) .{ }^{13} \mathrm{CNMR}(100 \mathrm{MHz}, \mathrm{DMSO}-d 6) \delta 163.42$ (C), 151.20 (C), $140.12(\mathrm{CH}), 138.39(\mathrm{C}), 132.31(\mathrm{CH}), 129.70(\mathrm{CH}), 129.26$ $(\mathrm{CH}), 127.21(\mathrm{CH}), 124.66(\mathrm{C}), 108.74(\mathrm{C}), 84.73(\mathrm{CH}), 63.44$ $\left(\mathrm{CH}_{2}\right), 36.43(\mathrm{C}), 26.52\left(\mathrm{CH}_{3}\right)$.

\section{5-[1-(phenyl)cyclohexyloxymethyl]uracil (10b)}

Treatment of $2(50 \mathrm{mg}, 0.311 \mathrm{mmol})$ with $290 \mathrm{mg}(1.52 \mathrm{mmol})$ of racemic a-(cyclohexyl)benzyl alcohol (1-phenylcyclohexanol) for 1 hour afforded after purification $20 \mathrm{mg}$ of product (20\%). ' H NMR (400 MHz, DMSO-d6) $\delta 11.08$ (br. s, $1 \mathrm{H}, \mathrm{D}_{2} \mathrm{O}$ exchangeable) 10.78 (br. s, $1 \mathrm{H}, \mathrm{D}_{2} \mathrm{O}$ exchangeable), 7.28 (m, $6 \mathrm{H}), 4.05(\mathrm{~d}, 1 \mathrm{H}, J=6.0 \mathrm{~Hz}), 3.93(\mathrm{AB} \mathrm{d}, 1 \mathrm{H}, J=12.0 \mathrm{~Hz}), 3.83$ $(A B d, 1 \mathrm{H}, J=12.0 \mathrm{~Hz}), 1.90(\mathrm{~m}, 1 \mathrm{H}), 1.69(\mathrm{~m}, 4 \mathrm{H}), 0.92(\mathrm{~m}, 6 \mathrm{H})$. ${ }^{13} \mathrm{CNMR}(100 \mathrm{MHz}$, DMSO-d6) $\delta 163.57$ (C), 151.21 (C), 140.95 $(\mathrm{CH}), 139.67(\mathrm{C}), 127.98(\mathrm{CH}), 127.20(\mathrm{CH}), 127.16(\mathrm{CH}), 108.74$ (C), 85.27 (CH), $62.79\left(\mathrm{CH}_{2}\right), 43.69(\mathrm{CH}), 28.75\left(\mathrm{CH}_{2}\right), 26.04\left(\mathrm{CH}_{2}\right)$, $25.41\left(\mathrm{CH}_{2}\right)$.

\section{5-[1-(2-nitrophenyl)cyclohexyloxymethyl]uracil (11b)}

Treatment of $2(50 \mathrm{mg}, 0.311 \mathrm{mmol})$ with $290 \mathrm{mg}(1.5 \mathrm{mmol})$ of racemic a-cyclohexyl-2-nitrobenzyl alcohol (1-(2-nitrophenyl) cyclohexanol) for 1 hour afforded after purification $20 \mathrm{mg}$ of product (20\%). ${ }^{1} H$ NMR (400 MHz, DMSO-d6) $\delta 11.08$ (br. s, 1 $\mathrm{H}, \mathrm{D}_{2} \mathrm{O}$ exchangeable) 10.83 (br. $\mathrm{s}, 1 \mathrm{H}, \mathrm{D}_{2} \mathrm{O}$ exchangeable), $7.92(\mathrm{~d}, 1 \mathrm{H}, J=9.1 \mathrm{~Hz}), 7.72(\mathrm{~m}, 1 \mathrm{H}), 7.72(\mathrm{~m}, 1 \mathrm{H}), 7.65(\mathrm{~m}, 1 \mathrm{H})$ $7.54(\mathrm{~m}, 1 \mathrm{H}), 7.37(\mathrm{~s}, 1 \mathrm{H}), 4.62(\mathrm{~d}, 1 \mathrm{H}, J=6.0 \mathrm{~Hz}), 3.97(\mathrm{AB} \mathrm{d}, 1$ $\mathrm{H}, J=11.7 \mathrm{~Hz}), 3.87(\mathrm{AB} \mathrm{d}, 1 \mathrm{H}, J=11.7 \mathrm{~Hz}), 1.65(\mathrm{~m}, 5 \mathrm{H}), 1.23$ $(\mathrm{m}, 1 \mathrm{H}), 1.07(\mathrm{~m}, 1 \mathrm{H}) .{ }^{13} \mathrm{C} N M R(100 \mathrm{MHz}$, DMSO-d6) $\delta 163.60$ (C), $151.18(\mathrm{C}), 149.10(\mathrm{C}), 140.71(\mathrm{CH}), 135.72(\mathrm{C}), 132.89(\mathrm{CH})$, $129.02(\mathrm{CH}), 128.47(\mathrm{CH}), 123.82(\mathrm{CH}), 108.79(\mathrm{C}), 79.57(\mathrm{CH})$, $63.56\left(\mathrm{CH}_{2}\right), 43.68(\mathrm{CH}), 28.99\left(\mathrm{CH}_{2}\right), 27.65\left(\mathrm{CH}_{2}\right), 25.86\left(\mathrm{CH}_{2}\right)$, $25.65\left(\mathrm{CH}_{2}\right), 25.43\left(\mathrm{CH}_{2}\right)$.

\section{5-[1-(phenyl)cyclohexyloxymethyl] uracil (12b)}

Treatment of 2 (50mg, $0.311 \mathrm{mmol})$ with $345 \mathrm{mg}(1.8 \mathrm{mmol})$ of racemic a-n-hexylbenzyl alcohol (1-phenylheptanol) for 2 hours afforded after purification $19 \mathrm{mg}$ of product $(19 \%) .{ }^{.} \mathrm{H}$ NMR (400 MHz, DMSO-d6) $\delta 11.08$ (br. s, $1 \mathrm{H}, \mathrm{D}_{2} \mathrm{O}$ exchangeable) 10.78 (br. s, $1 \mathrm{H}, \mathrm{D}_{2} \mathrm{O}$ exchangeable), $7.31(\mathrm{~m}, 6 \mathrm{H}), 4.32$ $(\mathrm{m}, 1 \mathrm{H}), 3.94(\mathrm{AB} \mathrm{d}, 1 \mathrm{H}, J=12.0 \mathrm{~Hz}), 3.89(\mathrm{AB} \mathrm{d}, 1 \mathrm{H}, J=12.0$ $\mathrm{Hz}), 1.67(\mathrm{~m}, 1 \mathrm{H}), 1.53(\mathrm{~m}, 1 \mathrm{H}), 1.22(\mathrm{~m}, 8 \mathrm{H}), 0.83(\mathrm{~m}, 3 \mathrm{H})$.

\section{5-[1-phenyl-3,3-(dimethyl)butoxymethyl] uracil(14b)}

Treatment of $2(50 \mathrm{mg}, 0.311 \mathrm{mmol})$ with $121 \mathrm{mg}(0.702 \mathrm{mmol})$ of a-neo-pentylbenzyl alcohol (3,3-dimethyl-1-phenyl-1-propanol, was obtained by Grignard addition of phenylmagnesium chloride to 3,3-dimethylbutanal) for 3 hours afforded after purification $6 \mathrm{mg}$ of product (6\%). ${ }^{1} \mathrm{HNMR}\left(400 \mathrm{MHz}, \mathrm{CD}_{3} \mathrm{OD}\right)$ $\delta 7.31(\mathrm{br} \mathrm{m}, 6 \mathrm{H}), 4.53(\mathrm{dd}, 1 \mathrm{H}, J=8.8,3.3 \mathrm{~Hz}), 4.00(\mathrm{~s}, 2 \mathrm{H})$,
1.81 (dd, $1 \mathrm{H}, J=14.5,8.8 \mathrm{~Hz}), 1.44(\mathrm{dd}, 1 \mathrm{H}, J=14.5,3.3 \mathrm{~Hz}), 0.97$ $(\mathrm{s}, 9)$. HRMS (ESI) for [MNa] ${ }^{+}$calculated: 325.15226 , observed: 325.15232

\section{5-[1-phenyl-3,3-(dimethyl)butoxymethyl]uracil(15b)}

Treatment of $2(50 \mathrm{mg}, 0.311 \mathrm{mmol})$ with $120 \mathrm{mg}(0.652 \mathrm{mmol})$ of diphenylmethanol for 3 hours afforded after purification $10 \mathrm{mg}$ of product (10\%). ${ }^{1} \mathrm{H} N \mathrm{NMR}(400 \mathrm{MHz}, \mathrm{DMSO}-\mathrm{d} 6) \delta 11.14$ (br. s, $\mathrm{D}_{2} \mathrm{O}$ exchangeable) 10.86 (br. s, $\mathrm{D}_{2} \mathrm{O}$ exchangeable), $7.37(\mathrm{~m}, 10 \mathrm{H}), 5.54(\mathrm{~s}, 1 \mathrm{H}), 4.10(\mathrm{~s}, 2 \mathrm{H})$. HRMS (ESI) $\left[\mathrm{MH}_{2} \mathrm{O}\right]^{+}$ calculated: 326.14992 , observed: 326.14989 ; for $[\mathrm{MNa}]^{+}$calculated: 331.10531, observed: 331.10536; for [M-H] calculated: 307.10882, observed: 307.10872.

\section{5-[1-phenyl-2-(methyl)propoxymethyl] uracil (16b)}

Treatment of $2(50 \mathrm{mg}, 0.311 \mathrm{mmol})$ with $467 \mathrm{mg}(3.114 \mathrm{mmol})$ of commercial racemic a-isopropylbenzyl alcohol (2-methyl1-phenyl-1-propanol) for 0.5 hours afforded after purification $60 \mathrm{mg}$ of product (70\%). ${ }^{~} \mathrm{H} N \mathrm{HR}(400 \mathrm{MHz}, \mathrm{DMSO}-\mathrm{d \sigma}) \delta 11.10$ (br. s, $\mathrm{D}_{2} \mathrm{O}$ exchangeable) 10.83 (br. s, $\mathrm{D}_{2} \mathrm{O}$ exchangeable), 7.28 $(\mathrm{m}, 6 \mathrm{H}), 4.03(\mathrm{AB} \mathrm{d}, 1 \mathrm{H}, J=6.9 \mathrm{~Hz}), 3.95(\mathrm{AB} \mathrm{d}, 2 \mathrm{H}, J=12.0 \mathrm{~Hz})$, $3.86(\mathrm{AB} \mathrm{d}, 2 \mathrm{H}, \mathrm{J}=12.0 \mathrm{~Hz}), 1.84(\mathrm{~m}, 1 \mathrm{H}), 0.89(\mathrm{AB} \mathrm{d}, 3 \mathrm{H}, \mathrm{J}=$ $6.5 \mathrm{~Hz}) 0.68(\mathrm{AB} \mathrm{d}, 3 \mathrm{H}, \mathrm{J}=6.5 \mathrm{~Hz}) .{ }^{13} \mathrm{C} \mathrm{NMR}\left(100 \mathrm{MHz}, \mathrm{CD}_{3} \mathrm{OD}\right)$ $\delta 164.08(\mathrm{C}), 151.71(\mathrm{C}), 141.50(\mathrm{C}), 140.16(\mathrm{CH}), 128.46(\mathrm{CH})$, $127.70(\mathrm{CH}), 127.62(\mathrm{CH}), 109.93(\mathrm{C}), 86.55(\mathrm{CH}), 63.40\left(\mathrm{CH}_{2}\right)$, $34.60(\mathrm{CH}), 19.27\left(\mathrm{CH}_{3}\right), 19.03\left(\mathrm{CH}_{3}\right)$. HRMS (ESI) for $[\mathrm{MNa}]^{+}$ calculated: 297.12096, observed: 297.12102.

\section{5-[1-phenyl-2-(methyl)propoxymethyl] uracil (18b)}

Treatment of $2(50 \mathrm{mg}, 0.311 \mathrm{mmol})$ with $384 \mathrm{mg}(3.144 \mathrm{mmol})$ of commercial racemic a-methylbenzyl alcohol (1-phenylethanol) for 3 hours afforded after purification $59 \mathrm{mg}$ of product (78\%). ${ }^{1} H \mathrm{NMR}(400 \mathrm{MHz}, \mathrm{DMSO}-\mathrm{d} 6) \delta 11.11$ (br. s, $\mathrm{D}_{2} \mathrm{O}$ exchangeable) 10.82 (br. $\mathrm{s}, \mathrm{D}_{2} \mathrm{O}$ exchangeable), 7.30 ( $\mathrm{m}$, $6 \mathrm{H}), 4.53(\mathrm{~m} 1 \mathrm{H}), 3.97(\mathrm{AB} \mathrm{d}, 2 \mathrm{H}, J=12.0 \mathrm{~Hz}), 3.86(\mathrm{AB} \mathrm{d}, 2$ $\mathrm{H}, J=12.0 \mathrm{~Hz}), 1.84(\mathrm{~m}, 1 \mathrm{H}), 1.33(\mathrm{~d}, 3 \mathrm{H}, \mathrm{J}=6.5 \mathrm{~Hz}) .{ }^{13} \mathrm{C} N M R$ $\left(100 \mathrm{MHz}, C D_{3} \mathrm{OD}\right) \delta 163.68$ (C), 151.24 (C), 143.59 (C), 139.93 $(\mathrm{CH}), 128.29(\mathrm{CH}), 127.24(\mathrm{CH}), 125.94(\mathrm{CH}), 109.43(\mathrm{C}), 76.65$ $(\mathrm{CH}), 62.44\left(\mathrm{CH}_{2}\right), 23.74\left(\mathrm{CH}_{3}\right)$. HRMS (ESI) for $[\mathrm{MNa}]^{+}$calculated: 269.08966, observed: 269.08966; for [M-H] calculated: 245.09317, observed: 245.09312.

\section{Quantitative PCR analysis}

Human embryonic kidney cells $\mathrm{HEK}_{293}$ were infected with human papilloma (strain: $\mathrm{HPV}_{11}$ ), human foreskin fibroblast HFF cells with BK (strain: Gardner), and human embryonic kidney 293TT cells with John Cunningham (strain: $M A_{-1}$ ) viruses. The infected cells were treated with DMSO solutions of test compounds and cidofovir, a known anti-viral drug ( ${ }^{\circ}$ Vistide), as positive control at concentrations $0,0.1,1,10,50,100,200$, and $300 \mu \mathrm{M}$. The cells were incubated for 72 hours. After incubation, the cells were harvested and their DNA was isolated followed by quantitative real-time PCR analyses using FastStart Universal Probe Master (ROX) (Roche Applied Science). PCR 
Borland et al. Biochemical Compounds 2016,

was performed with an initial denaturation reaction at $95^{\circ} \mathrm{C}$ for 1 minute and then amplified with 40 cycles of $95^{\circ} \mathrm{C}$ for 30 seconds, $60^{\circ} \mathrm{C}$ for 30 seconds, and $72^{\circ} \mathrm{C}$ for 30 seconds. The amplification was monitored on Step One Plus (Applied Biosystems Inc.). A $5 \mu \mathrm{L}$ aliquot of the resulting solution was loaded into each well containing a $12 \%$ denaturing PAGE gel, which was subsequently run at constant 18 Watts for 35 minutes. The gel was visualized using the Odyssey Infrared Imaging System (LiCor) with $169 \mu \mathrm{m}$ resolution and the 700-channel laser source which has a solid-state laser diode at $680 \mathrm{~nm}$ and ImageQuant 5.0 software was used to determine density measurements. Experiments were performed in triplicate. $\mathrm{EC}_{50}$ and $\mathrm{EC}_{90}$ values were determined by comparison to the vehicle (negative control). The concentrations of compounds causing $50 \%$ and $90 \%$ inhibition of HPV and JCC viral DNA replication are summarized in Tables $\mathbf{1}$ and 2, respectively.

\section{Cell viability}

The CellTiter-Glo ${ }^{\otimes}$ Luminescent Cell Viability Assay was used. Upon incubation, cells were treated with solution of beetle luciferin in the presence of ATP. Luminescence was recorded $10 \mathrm{~min}$ utes after reagent addition using a GloMax ${ }^{\oplus}$-Multi+Detection System. The luminescent signal from the host cells was compared to the background signal from serum-supplemented medium without cells. The $\mathrm{CC}_{50}$ curves were determined by plotting viability versus compound concentration. Kaleidagraph software was used to calculate the $R$ value for each logarithmic curve fitting. The results are outlined in Tables $\mathbf{1}$ and $\mathbf{2}$.

Table 1. Inhibition of human papillomavirus DNA replication $\left(\mathrm{EC}_{50}, \mathrm{EC}_{90}\right)$, toxicity in human host cells $\left(\mathrm{CC}_{50}\right)$ and breast cancer cells $\left(\mathrm{IC}_{50}\right)[27,29]$.

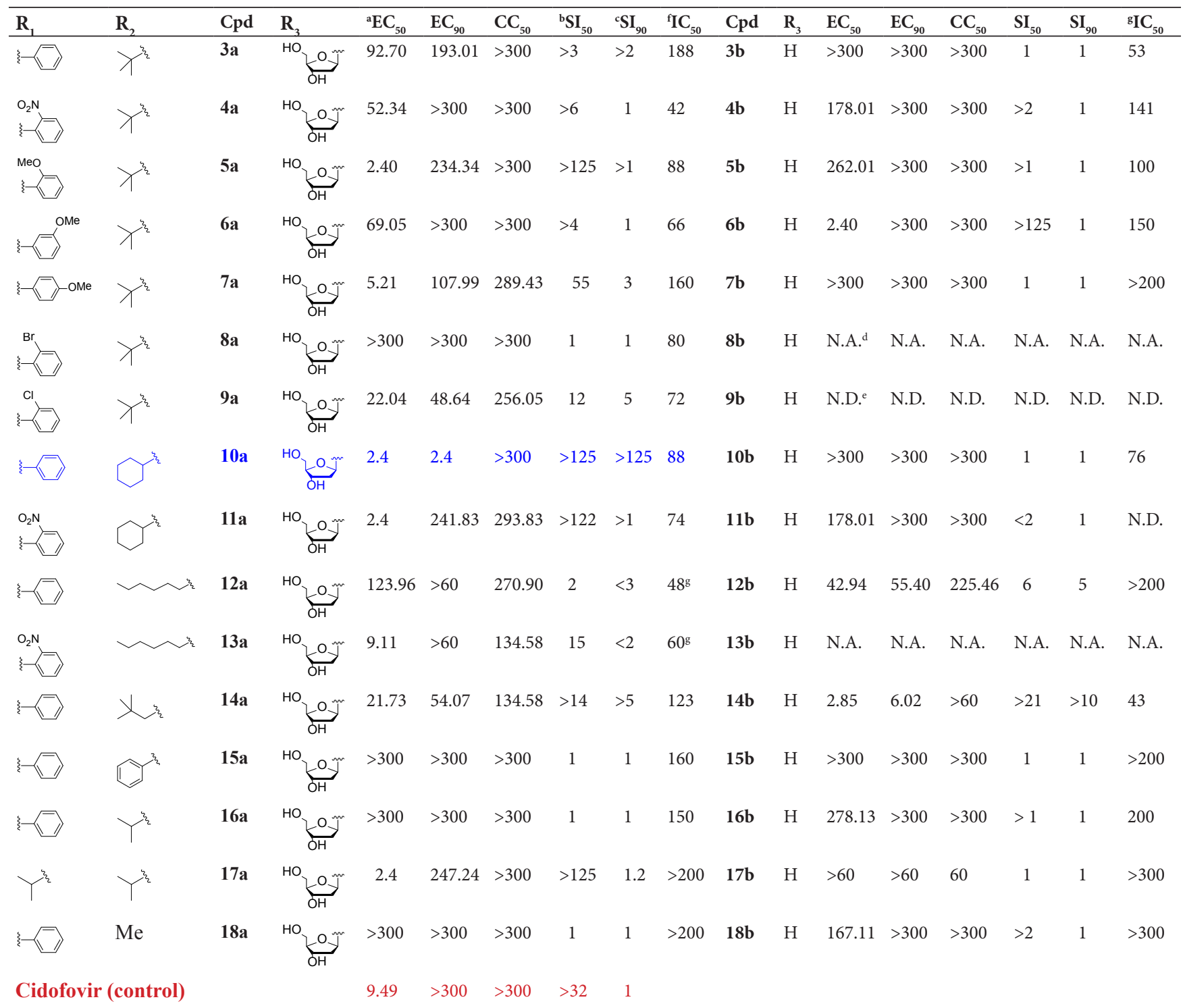

${ }^{\mathrm{a}}$ All concentrations are in $\mu \mathrm{M} ;{ }^{\mathrm{b}} \mathrm{SI}_{50}=\mathrm{CC}_{50} / \mathrm{EC}_{50} ;{ }^{\mathrm{c}} \mathrm{SI}_{90}=\mathrm{CC}_{50} / \mathrm{EC}_{90}$; ${ }^{\mathrm{d}} \mathrm{Cpd}$ could not be synthesized; ${ }^{\mathrm{e}} \mathrm{Cpd}$ was not tested ${ }^{\mathrm{f}} \mathrm{Ref} .27 ;{ }^{\mathrm{g}} \mathrm{Ref} .29$. 
Borland et al. Biochemical Compounds 2016,

http://www.hoajonline.com/journals/pdf/2052-9341-4-3.pdf

doi: 10.7243/2052-9341-4-3

Table 2. Inhibition of John Cunningham virus DNA replication $\left(\mathrm{EC}_{50}, \mathrm{EC}_{90}\right)$ and toxicity in human host cells $\left(\mathrm{CC}_{50}\right)$.

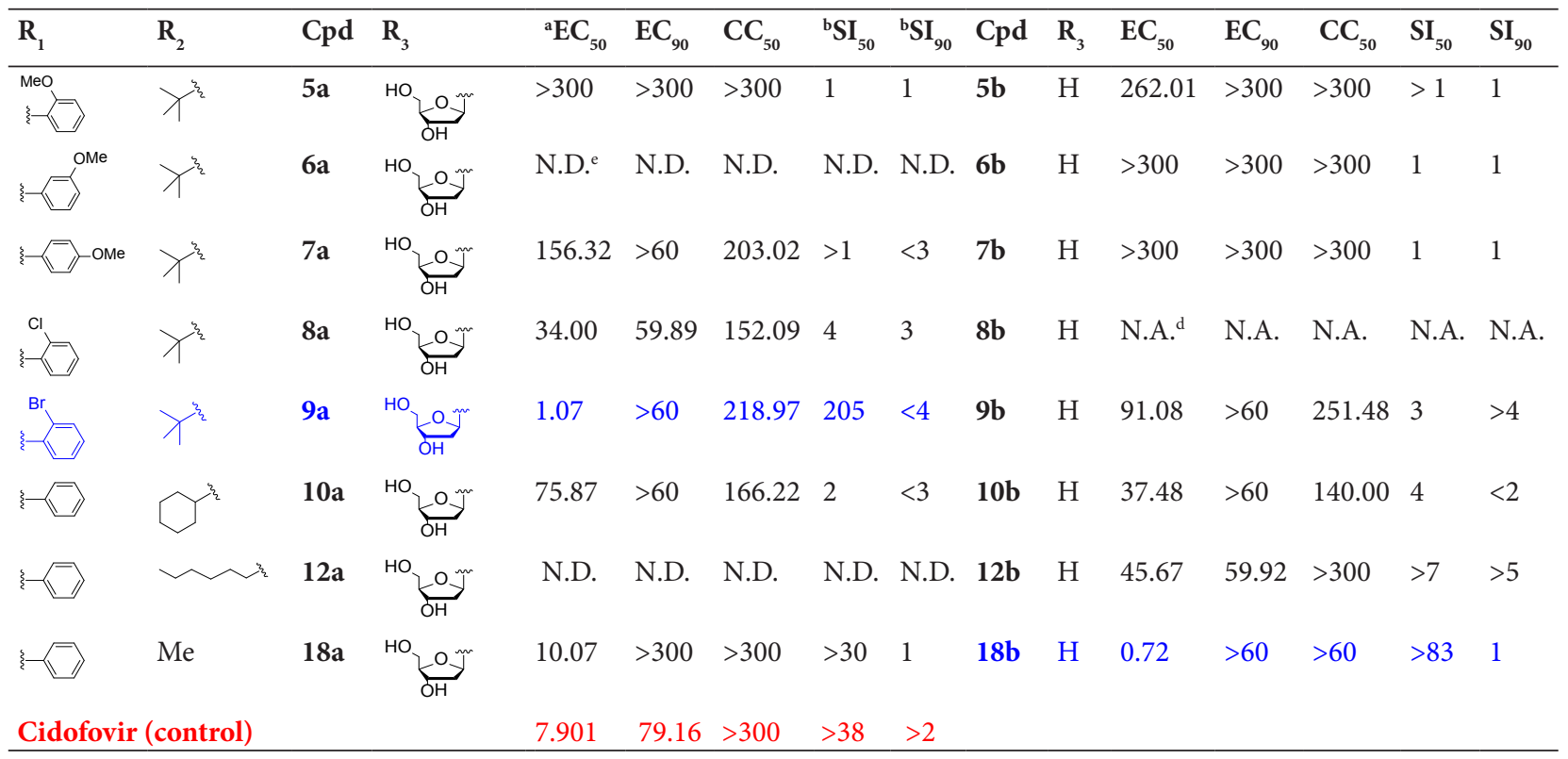

${ }^{\mathrm{a}}$ All concentrations are in $\mu \mathrm{M} ;{ }^{\mathrm{b}} \mathrm{SI}_{50}=\mathrm{CC}_{50} / \mathrm{EC}_{50} ;{ }^{\mathrm{c}} \mathrm{SI}_{90}=\mathrm{CC}_{50} / \mathrm{EC}_{90}$; ${ }^{\mathrm{d}} \mathrm{Cpd}$ could not be synthesized; ${ }^{\mathrm{e}} \mathrm{Cpd}$ was not tested.

\section{Results and discussion}

The bioactive compounds were obtained by heating 5-bromomethyl-3-N-(tert-butyloxy)carbobyl-3,'5'-bis-(tert-butyl) dimethylsilyl-O-2'-deoxyuridine (1) [28] or 5-chloromethyluracil (2) with an appropriate alcohol under neat, anhydrous conditions, as reported previously [27]. This transformation yielded nucleobases $\mathbf{3 b - 1 8 b}$; removal of the residual TBS groups using tetra- $n$-butylammonium fluoride yielded nucleoside derivatives 3a-18a (Figure 1). The PCR DNA and CellTiter-Glo assays were performed using standard protocols.

The data reflecting quantitative inhibition of human papilloma virus replication is summarized in Table 1. The trends revealed by structure-activity relationship are quite different from those observed for cytotoxicity toward cancer cells [27]. First, the a-tert-butyl and 2-nitrobenzyl groups were not the best substituents, as evidenced by the higher $\mathrm{EC}_{50}$ of $\mathbf{3 a}$ compared to $10 \mathbf{a}$, as well as $4 \mathbf{a}$ versus $\mathbf{1 1} \mathbf{a}$, and $\mathbf{4 a}$ versus $\mathbf{5 a}$. Furthermore, when the methoxy group in the benzyl substituent in 5a was moved across the ring, the activity of the meta-substituted analog (6a) was decreased, while restored for the para-substituted analog $7 \mathbf{a}$, which is the opposite of the anti-cancer activity trend [27]. Second, the presence of an aryl group as either $R_{1}$ or $R_{2}$ was not critical, as evidenced by the high activity of 16a. And third, modified nucleobases demonstrated superior activity compared to the corresponding base-modified nucleosides in greater number of instances, particularly, $\mathbf{6 b}$ compared to $\mathbf{6} \mathbf{a}, \mathbf{1 2 b}$ to $\mathbf{1 2 a}$, and $\mathbf{1 4 b}$ to $\mathbf{1 4 a}$. The most impressive potency, however, was demonstrated by the nucleoside derivative 10a, 5-(a-cyclohexylbenzyloxy) methyl-2'-deoxyuridine, with the $90 \%$ efficiency $\left(\mathrm{EC}_{90}\right)$ value of $2.4 \mu \mathrm{M}$ and the $50 \%$ cytotoxicity $\left(\mathrm{CC}_{50}\right)$ value exceeding $300 \mu \mathrm{M}$. The outstanding $\mathrm{SI}_{90}$ value of over 125 makes 10a a definite hit compound for further drug development, given significant side effects of cidofovir [30,31] whose potency is lower. Expectedly, cytotoxicity of almost all the examined antimetabolites toward host cells is significantly lower than that toward breast cancer cells [29].

The trend discovered for inhibition of JC viral DNA replication was somewhat different (Table 2 ). The three most active compounds turned out to be 5-(a-tert-butylortho-bromobenzyloxy) methyl-2'-deoxyuridine $\mathbf{9 a}$ and 5-(a-methylbenzyloxy)methyluracil $\mathbf{1 8 b}$, showing, respectively, moderate and low activity against HPV DNA replication (Table 1). Notably, nucleoside 18a showed 14 fold lower potency compared to the nucleobase, which is unprecedented in the anti-cancer activity of this type of species $[27,29]$. Surprisingly, the chloro-analog of $\mathbf{9 a}$, compound $\mathbf{8} \mathbf{a}$, was only somewhat active, and the activity of $\mathbf{1 0 a}$, the lead compound for HPV, was rather modest against JCV. Neither of these nucleoside and nucleobase derivatives significantly inhibited DNA replication of the BK virus (see supporting information).

\section{Conclusions}

We have examined the activity of 5-substituted thymidine and thymine derivatives with respect to inhibition of DNA replication in human papilloma, John Cunningham, and BK viruses. Studies of the structure-activity relationship revealed hits for HPV and JCV, but none against BKV. Importantly, these bioactive species with high anti-viral activity have low cytotoxicity, which makes them novel lead compounds with the 
Borland et al. Biochemical Compounds 2016,

http://www.hoajonline.com/journals/pdf/2052-9341-4-3.pdf

doi: $10.7243 / 2052-9341-4-3$

potential to pursue further drug development.

\section{Supporting information}

Spectral characterization of novel molecules, NIAID-

$\mathrm{NIH}$ in-vitro antiviral screening reports, and the original

$248^{\text {th }}$ ACS National Meeting poster [29]. This material is available free of charge at Supplementary data.

\section{Competing interests}

The authors declare that they have no competing interests.

Authors' contributions

\begin{tabular}{|l|c|c|c|c|c|c|c|c|}
\hline $\begin{array}{l}\text { Authors' } \\
\text { contributions }\end{array}$ & KMB & PRW & MPB & SML & CAS & APB & JNT & VAL \\
\hline $\begin{array}{l}\text { Research concept } \\
\text { and design }\end{array}$ & $\checkmark$ & -- & -- & -- & -- & -- & -- & $\checkmark$ \\
\hline $\begin{array}{l}\text { Collection and/or } \\
\text { assembly of data }\end{array}$ & $\checkmark$ & $\checkmark$ & $\checkmark$ & $\checkmark$ & $\checkmark$ & $\checkmark$ & $\checkmark$ & $\checkmark$ \\
\hline $\begin{array}{l}\text { Data analysis and } \\
\text { interpretation }\end{array}$ & $\checkmark$ & -- & -- & -- & -- & -- & -- & $\checkmark$ \\
\hline Writing the article & -- & -- & -- & -- & -- & -- & -- & $\checkmark$ \\
\hline $\begin{array}{l}\text { Critical revision of } \\
\text { the article }\end{array}$ & -- & -- & -- & -- & -- & -- & -- & $\checkmark$ \\
\hline $\begin{array}{l}\text { Final approval of } \\
\text { article }\end{array}$ & -- & -- & -- & -- & -- & -- & -- & $\checkmark$ \\
\hline Statistical analysis & -- & -- & -- & -- & -- & -- & -- & $\checkmark$ \\
\hline
\end{tabular}

\section{Acknowledgement}

This work was supported by the University of Cincinnati start-up and the University of Cincinnati Individual Faculty Research Grant funds to Prof. Litosh. We also gratefully acknowledge the US National Institute of Allergy and Infectious Diseases-National Institutes of Health (NIAID$\mathrm{NIH}$ ) for performing anti-viral screening through the Division of Microbiology and Infectious Diseases (DMID).

Publication history

Editor: Ajoy Basak, University of Ottawa, Canada.

EIC: Mariusz Skwarczynski, The University of Queensland, Australia. Received: 13-May-2016 Final Revised: 04-Jul-2016

Accepted: 11-Jul-2016 Published: 22-Jul-2016

\section{References}

1. Ramakrishnan S, Partricia S and Mathan G. Overview of high-risk HPV's 16 and 18 infected cervical cancer: pathogenesis to prevention. Biomed Pharmacother. 2015; 70:103-10. | Article | PubMed

2. Veldman T, Horikawa I, Barrett JC and Schlegel R. Transcriptional activation of the telomerase hTERT gene by human papillomavirus type 16 E6 oncoprotein. J Virol. 2001; 75:4467-72. | Article | PubMed Abstract | PubMed FullText

3. Bulut G, Fallen S, Beauchamp EM, Drebing LE, Sun J, Berry DL, Kallakury $B$, Crum CP, Toretsky JA, Schlegel $R$ and Uren A. Beta-catenin accelerates human papilloma virus type-16 mediated cervical carcinogenesis in transgenic mice. PLoS One. 2011; 6:e27243. | Article | PubMed Abstract I PubMed FullText

4. Stoppler H, Koval D and Schlegel R. The serine protease inhibitors TLCK and TPCK inhibit the in vitro immortalization of primary human keratinocytes by HPV-18 DNA. Oncogene. 1996; 13:1545-8. | Article | PubMed

5. Yuan H, Myers S, Wang J, Zhou D, Woo JA, Kallakury B, Ju A, Bazylewicz M, Carter YM, Albanese C, Grant N, Shad A, Dritschilo A, Liu X and Schlegel R. Use of reprogrammed cells to identify therapy for respiratory papillomatosis. N Engl J Med. 2012; 367:1220-7. | Article | PubMed Abstract | PubMed FullText
6. Padgett BL, Walker DL, ZuRhein GM, Eckroade RJ and Dessel BH. Cultivation of papova-like virus from human brain with progressive multifocal leucoencephalopathy. Lancet. 1971; 1:1257-60. | Article | PubMed

7. Fishman JA. BK virus nephropathy--polyomavirus adding insult to injury. N Engl J Med. 2002; 347:527-30. | Article | PubMed

8. Burke MP, Borland KM and Litosh VA. Base-Modified Nucleosides as Chemotherapeutic Agents: Past and Future. Curr Top Med Chem. 2016; 16:1231-41. | Article | PubMed

9. Tiwari M. Antimetabolites: established cancer therapy. $J$ Cancer Res Ther. 2012; 8:510-9. | Article | PubMed

10. Capasso $C$ and Supuran CT. Sulfa and trimethoprim-like drugs antimetabolites acting as carbonic anhydrase, dihydropteroate synthase and dihydrofolate reductase inhibitors. J Enzyme Inhib Med Chem. 2014; 29:379-87. | Article | PubMed

11. Eriksson S, Munch-Petersen B, Johansson K and Eklund H. Structure and function of cellular deoxyribonucleoside kinases. Cell Mol Life Sci. 2002; 59:1327-46. | Article | PubMed

12. Zhang J, Visser F, King KM, Baldwin SA, Young JD and Cass CE. The role of nucleoside transporters in cancer chemotherapy with nucleoside drugs. Cancer Metastasis Rev. 2007; 26:85-110. | Article | PubMed

13. Chen LS, Plunkett $W$ and Gandhi V. Polyadenylation inhibition by the triphosphates of deoxyadenosine analogues. Leuk Res. 2008; 32:157381. | Article | PubMed Abstract | PubMed FullText

14. Kuchta RD, Ilsley D, Kravig KD, Schubert S and Harris B. Inhibition of DNA primase and polymerase alpha by arabinofuranosylnucleoside triphosphates and related compounds. Biochemistry. 1992; 31:4720-8. I Article | PubMed

15. Parker WB, Shaddix SC, Chang CH, White EL, Rose LM, Brockman RW, Shortnacy AT, Montgomery JA, Secrist JA, 3rd and Bennett LL, Jr. Effects of 2-chloro-9-(2-deoxy-2-fluoro-beta-D-arabinofuranosyl)adenine on K562 cellular metabolism and the inhibition of human ribonucleotide reductase and DNA polymerases by its $\mathbf{5}^{\prime}$-triphosphate. Cancer Res. 1991; 51:2386-94. | Article | PubMed

16. Peters GJ, van der Wilt CL, van Groeningen CJ, Smid K, Meijer S and Pinedo HM. Thymidylate synthase inhibition after administration of fluorouracil with or without leucovorin in colon cancer patients: implications for treatment with fluorouracil. J Clin Oncol. 1994; 12:203542. | Article | PubMed

17. Hill DL and Bennett LL, Jr. Purification and properties of 5-phosphoribosyl pyrophosphate amidotransferase from adenocarcinoma 755 cells. Biochemistry. 1969; 8:122-30. | Article | PubMed

18. Genini D, Adachi S, Chao Q, Rose DW, Carrera CJ, Cottam HB, Carson DA and Leoni LM. Deoxyadenosine analogs induce programmed cell death in chronic lymphocytic leukemia cells by damaging the DNA and by directly affecting the mitochondria. Blood. 2000; 96:3537-43. | Article | PubMed

19. Fairchild CR, Maybaum J and Kennedy KA. Concurrent unilateral chromatid damage and DNA strand breakage in response to 6-thioguanine treatment. Biochem Pharmacol. 1986; 35:3533-41. | Article I PubMed

20. Marinkovic G, Kroon J, Hoogenboezem M, Hoeben KA, Ruiter MS, Kurakula K, Otermin Rubio I, Vos M, de Vries CJ, van Buul JD and de Waard V. Inhibition of GTPase Rac1 in endothelium by 6-mercaptopurine results in immunosuppression in nonimmune cells: new target for an old drug. J Immunol. 2014; 192:4370-8. | Article | PubMed

21. Swann PF, Waters TR, Moulton DC, Xu YZ, Zheng Q, Edwards M and Mace R. Role of postreplicative DNA mismatch repair in the cytotoxic action of thioguanine. Science. 1996; 273:1109-11. | Article | PubMed

22. Hostetler $K Y$, Stuhmiller $L M$, Lenting $H B$, van den Bosch $H$ and Richman DD. Synthesis and antiretroviral activity of phospholipid analogs of azidothymidine and other antiviral nucleosides. J Biol Chem. 1990; 265:6112-7. | Article | PubMed

23. Macias J, Neukam K, Mallolas J, Lopez-Cortes LF, Carton JA, Domingo P, Moreno S, Iribarren JA, Clotet B, Crespo M, de Los Santos I, Ortega E, 
Borland et al. Biochemical Compounds 2016,

http://www.hoajonline.com/journals/pdf/2052-9341-4-3.pdf

Knobel H, Jimenez-Exposito MJ and Pineda JA. Liver toxicity of initial antiretroviral drug regimens including two nucleoside analogs plus one non-nucleoside analog or one ritonavir-boosted protease inhibitor in HIV/HCV-coinfected patients. HIV Clin Trials. 2012; 13:61-9. | Article | PubMed

24. Momparler RL, Bouffard DY, Momparler LF, Dionne J, Belanger K and Ayoub J. Pilot phase I-II study on 5-aza-2'-deoxycytidine (Decitabine) in patients with metastatic lung cancer. Anticancer Drugs. 1997; 8:358-68. | Article | PubMed

25. Arce C, Segura-Pacheco B, Perez-Cardenas E, Taja-Chayeb L, Candelaria $M$ and Duennas-Gonzalez $A$. Hydralazine target: from blood vessels to the epigenome. J Trans/ Med. 2006; 4:10. | Article | PubMed Abstract | PubMed FullText

26. Dragovic $G$ and Jevtovic $D$. The role of nucleoside reverse transcriptase inhibitors usage in the incidence of hyperlactatemia and lactic acidosis in HIV/AIDS patients. Biomed Pharmacother. 2012; 66:308-11. | Article | PubMed

27. Borland KM, AbdulSalam SF, Solivio MJ, Burke MP, Wolfkiel PR, Lawson SM, Stockman CA, Andersen JM, Smith S, Tolstolutskaya JN, Gurjar PN, Bercz AP, Merino EJ and Litosh VA. Base-modified thymidines capable of terminating DNA synthesis are novel bioactive compounds with activity in cancer cells. Bioorg Med Chem. 2015; 23:1869-81. I Article I PubMed Abstract I PubMed FullText

28. Litosh VA, Wu W, Stupi BP, Wang J, Morris SE, Hersh MN and Metzker ML. Improved nucleotide selectivity and termination of 3'-OH unblocked reversible terminators by molecular tuning of 2-nitrobenzyl alkylated HOMedU triphosphates. Nucleic Acids Res. 2011; 39:e39. | Article | PubMed Abstract | PubMed FullText

29. Borland KM, Lawson SM, Ventura S, Merino EJ and Litosh VA. Novel modified nucleobases that show cytotoxicity towards breast cancer cells. Abstracts of Papers, 248th ACS Nat'l Meeting Exp., San Francisco, CA, United States, August 10-14, 2014, MEDI-469.

30. Broekema FI and Dikkers FG. Side-effects of cidofovir in the treatment of recurrent respiratory papillomatosis. Eur Arch Otorhinolaryngol. 2008; 265:871-9. | Article | PubMed Abstract | PubMed FullText

31. Soma MA and Albert DM. Cidofovir: to use or not to use? Curr Opin Otolaryngol Head Neck Surg. 2008; 16:86-90. | Article | PubMed

\section{Citation:}

Borland KM, Wolfkiel PR, Burke MP, Lawson SM, Stockman CA, Bercz AP, Tolstolutskaya JN and Litosh VA. Base-modified thymidine and thymine analogs with low cytotoxicity effectively obstruct DNA replication in papovaviridae. Bio Chem Comp. 2016; 4:3.

http://dx.doi.org/10.7243/2052-9341-4-3 